\title{
A COMPARATIVE STUDY BETWEEN LENSTAR LS 900 AND IOLMASTER 700 FOR TORIC INTRAOCULAR LENS POWER CALCULATION
}

Alaa ElDin ElZawawi, Nader Hussein Lotfy Bayoumi, Enduka Okoto Thomas

Department of Ophthalmology, Faculty of Medicine, University of Alexandria, Egypt.

\section{INTRODUCTION}

Cataract surgery evolved dramatically from a simple procedure of removing the clouded crystalline lens to a method that additionally aims to correct all refractive errors, allowing a perfect restoration of the visual function with high levels of spectacle independence.

Astigmatism is a meridian-dependent refractive error, which is found in most human eyes. A large proportion of patients with cataract have corneal astigmatism ranging from 0.75 to 2.00 diopters (D).

Nowadays, implanting toric intraocular lenses (IOLs) remain the most common procedure to treat pre-existing corneal astigmatism during phacoemulsification.

Ocular biometric measurements are crucial in the calculation of IOL power before cataract Ory be be optical low-coler advantage of measuring the Total keratometry using SS-OCT.

\section{AIM OF THE WORK}

The aim of this work is to compare the LENSTAR LS 900 and the IOLMASTER 700 in toric intraocular lens power calculation.

\section{SUBJECTS AND METHODS}

SUBJECTS: This study included 30 eyes of 30 patients who underwent uneventful phacoemulsifation surgery with implantation of toric IOL and with the following criteria: Patients with visually significant senile cataract, regular corneal astigmatism ranging from one to five diopters and axial length between 19 to 24 millimeters.

METHODS: This was conducted as a prospective interventional study on patients presenting to and operated upon in the Ophthalmology Department of Alexandria Main University Hospital (AMUH). All patients before enrollment in the study provided written informed consent.

The IOLMaster 700 and the LENSTAR LS 900 were used in each patient, on the same visit, to measure different parameters and calculate the toric IOL power using the Barrett formula. For the Lenstar LS 900, the T-cone was used to measure the anterior corneal astigmatism and for the IOLMaster 700, the total corneal astigmatism was measured based on SS-OCT.

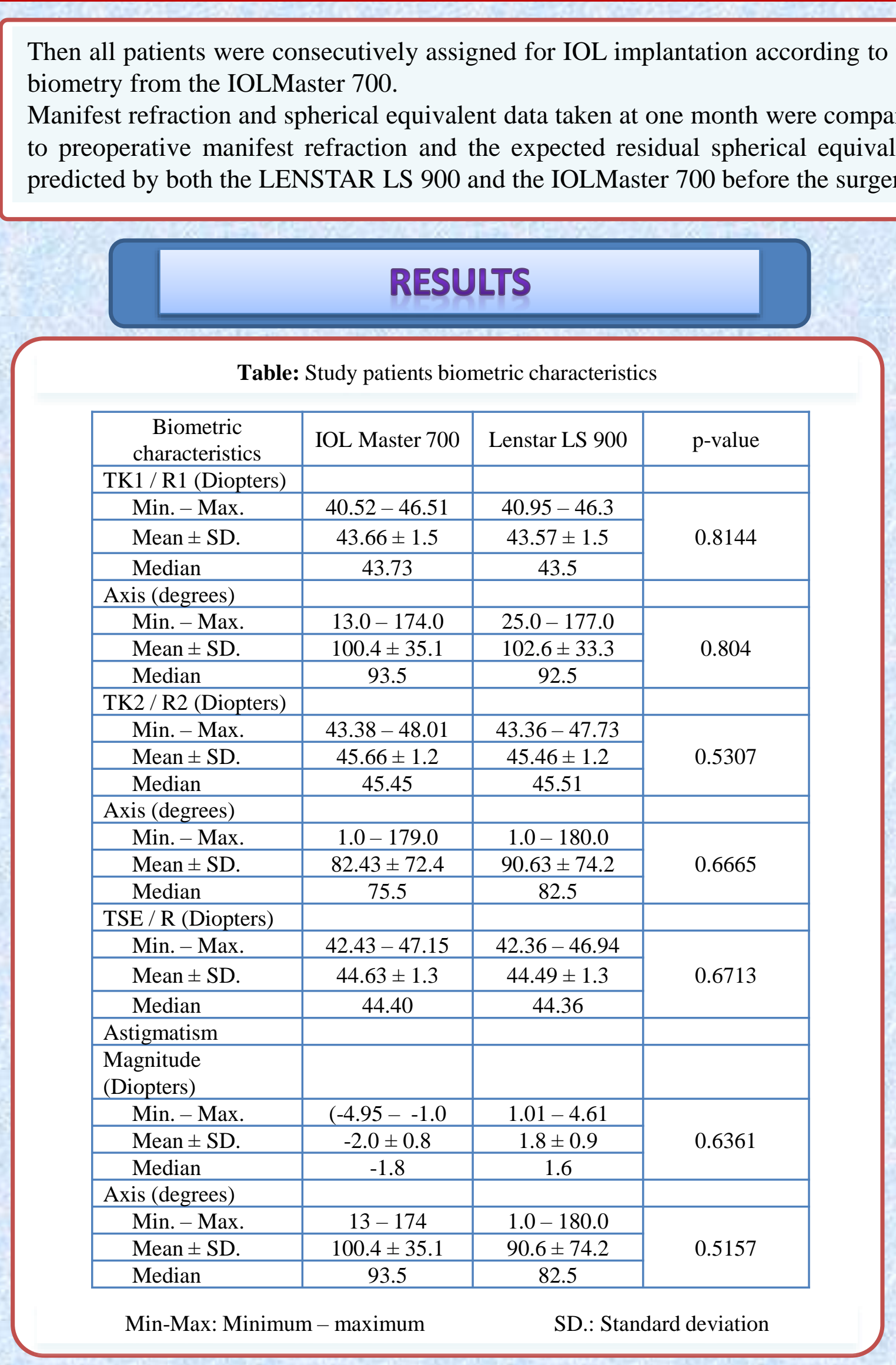

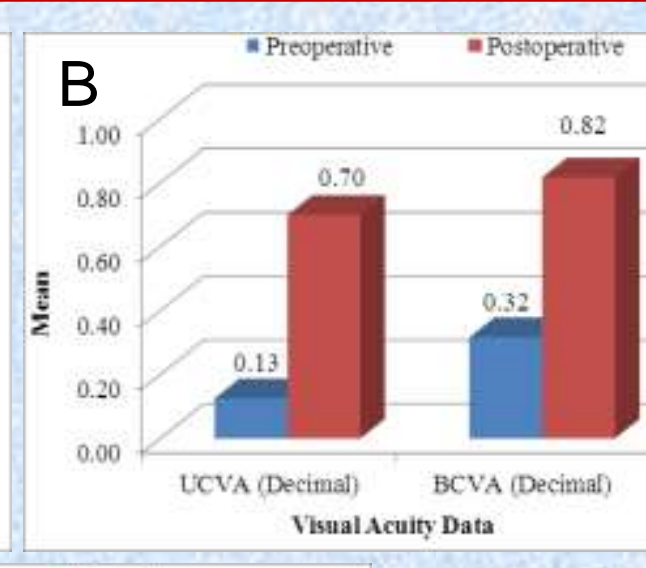

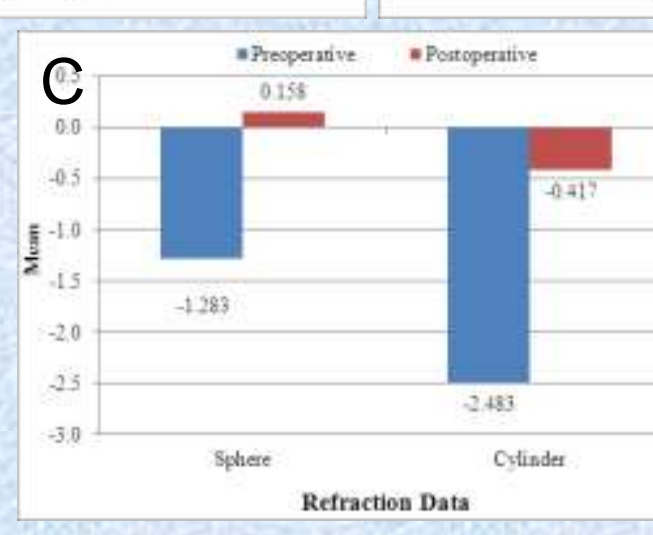

Figure (1): A) Comparison between the two machines according to Absolute Prediction Erro B) Comparison between the Preoperative and Postoperative visual acuity dat C) Comparison between the Preoperative and Postoperative refraction data.

\section{CONCLUSION}

- Optical rehabilitation by toric IOL implantation after phacoemulsification of senile cataract in eyes with corneal astigmatism is highly successful in achieving minimal postoperative refractive error and optimal postoperative visual acuity in operated eyes. The predictability of both the IOLMaster 700 and the Lenstar LS 900 for postoperative refraction of eyes implanted with toric IOLs is high and the two machines yield closely similar results.

There is no practical difference in the postoperative outcomes of toric IOL implantation for phacoemulsification of senile cataract in eyes with corneal astigmatism based on biometric calculations of either machines, the IOLMaster 700 and the Lenstar LS 900

2021 Alexandria Faculty of Medicine
CC-BY-NC

